Revista de Antropología Social

ISSN: 1131-558X

http://dx.doi.org/10.5209/RASO.59439

\title{
Diálogos interdisciplinares y debates actuales en los estudios sobre familia y parentesco
}

Grau Rebollo, Jorge. 2016. Nuevas formas de familia. Ámbitos emergentes. Barcelona: Ediciones Bellaterra.

Este libro se suma a la serie de publicaciones producidas desde el Grupo de Estudio Transcultural del Parentesco que integra el Grupo de Investigación en Antropología Fundamental y Orientada (GETP-GRAFO), en la Universidad Autónoma de Barcelona. A partir de investigaciones de campo llevadas a cabo dentro y fuera de España, el GETP-GRAFO ha contribuido desde su creación en 1994 a la reflexión sobre las teorías del parentesco y a la formulación de una propuesta teórica basada en un abordaje comparativo y transcultural del dominio analítico del parentesco. Jorge Grau Rebollo, miembro de este equipo de trabajo, invita en su nuevo libro a introducirnos en los cambios recientes operados en el ámbito de la familia y los grupos domésticos a través del caso del Estado español, señalando las principales problemáticas y abordajes que se han propuesto desde el campo de las ciencias sociales y humanas.

Luego de las críticas de Rodney Needham (1971) y David Schneider (1984) a las teorías clásicas del parentesco, la antropología quedó situada frente a dos alternativas: focalizar el estudio de las transformaciones en el modelo cultural del parentesco occidental o responder al desafío de elaborar nuevas perspectivas transculturales que devolvieran al parentesco su carácter universal y comparativo (González Echevarría, 2010). El libro, efectivamente, se centra en los cambios acaecidos en nuestras sociedades en los últimos 30 años y en la vasta y variada producción académica concomitante; no obstante, por otro lado, su interés reside en identificar los nudos temáticos y el universo de fenómenos bajo estudio que organizan un debate en el que confluyen distintos abordajes disciplinares, teóricos y metodológicos. Y en ese sentido, el ejercicio analítico de dar orden a la proliferación de publicaciones aporta a la reflexión y formulación de planteamientos teóricos y comparativos más generales que, aun quedando dentro del campo del parentesco folk, trascienden los estudios de caso particulares de los que se nutre.

Resulta una obra de gran utilidad, especialmente, para aquellos investigadores que se inician en este terreno de investigación así como para un público de estudiantes de grado y posgrado; les permite en ambos casos aproximarse con actualidad a problemáticas clásicas de la antropología del parentesco. Este carácter introductorio no invalida el trasfondo de un arduo trabajo erudito de organización, síntesis y clasificación de la extensa producción académica proveniente de la antropología, la sociología, la demografía y la historia. Su interés no reside en ofrecer un recuento exhaustivo de los aportes que desde el siglo XIX han realizado las diferentes disciplinas, sino en identificar algunos de los ejes temáticos más destacados que orientan los estudios más recientes sobre la familia y los nuevos grupos domésticos. No obstante, como el autor señala, no siempre se trata de la emergencia de fenómenos no- 
vedosos; en muchas ocasiones, nos enfrentamos a los mismos hechos pero dotados de mayor visibilidad y sobre los cuales se han desarrollado nuevas aproximaciones teóricas, especialmente a partir de la década de 1980.

La introducción se ocupa de señalar estos núcleos problemáticos: 1) la diversificación de las formas de unión afectiva y sexual y las múltiples combinaciones posibles que surgen de la identidad de género y la elección sexual de los individuos procreadores, factores que ponen en cuestión el modelo "tradicional" de familia monogamia, heterosexualidad de los miembros de la pareja y división sexual del trabajo- - Si el peso antes estaba en el matrimonio, ahora reside en la procreación y las relaciones paterno-filiales, constituyendo la progenie el "pivote relacional" que permite la articulación de diferentes roles y estrategias de quienes están encargados de su cuidado, función a la que también aportan personas ajenas al núcleo conyugal como parientes y amigos. 2) Los modos en que nuevos individuos son incorporados al núcleo procreativo básico; las nuevas tecnologías de reproducción humana asistida, la maternidad subrogada así como la adopción internacional señalan otras alternativas más allá de la descendencia directa por vía sexual. 3) La crianza ocupa un lugar central en los cambios que están operando en la familia y en este punto focalizan algunas investigaciones, aunque probablemente el énfasis que el autor le otorga también se deba a la propia perspectiva teórica del GETP-GRAFO que reconoce en la crianza un elemento clave y orientador de la definición del dominio analítico del parentesco. 4) El rol de las mujeres en el universo de las relaciones personales, especialmente en relación con los cambios vinculados a su dimensión profesional y a los roles domésticos asignados. 5) Las implicancias de la movilidad geográfica y la articulación de redes parentales que contribuyen a la crianza de los niños.

Un primer capítulo realiza un trazado histórico de los estudios sobre la familia en función de los temas y debates actuales que recorta el libro, proveyendo así el contexto sociohistórico en el que las cinco problemáticas mencionadas fueron formuladas y cobraron visibilidad. Luego, la obra se organiza en otros 7 capítulos que exploran la emergencia de nuevas prácticas y realidades familiares que involucran nuevas formas de relacionalidad, crianza y convivencia. Las familias reconstituidas y la monoparentalidad; la nuevas tecnologías de reproducción asistida; la convivencia no conyugal y las parejas living apart together — "vivir separadamente juntos"-; la elección sexual de los miembros de la pareja y la organización de la crianza; los matrimonios entre individuos de diferentes etnias o nacionalidades y los procesos migratorios; la circulación de menores y la adopción internacional; y las parejas de doble ingreso y sin hijos, son los fenómenos a través de los cuales el autor mapea la literatura y los enfoques actuales. Si bien el lector podría esperar que los 5 ejes temáticos reseñados fueran los criterios que establecieran la división por capítulos, esto no es así; la estructura de la obra se basa en la identificación de "las principales formas emergentes de relacionalidad, crianza y convivencia" en las cuales dichos núcleos se expresan (pág. 21). Grau Rebollo avanza sugiriendo, en cada capítulo, vinculaciones entre los fenómenos empíricos y las problemáticas que atraviesan los diferentes enfoques y campos disciplinares, pero corresponde aquí al lector profundizar en ese ejercicio de reflexión, obligando a un diálogo entre la Introducción y el capítulo 1 con el resto del libro. En ese mismo sentido, llama la atención la ausencia de un epílogo en donde el autor retome las orientaciones analíticas que anticipó al comienzo de la obra; esto reforzaría su propio abordaje y ayudaría al lector a reponer bajo una mirada más integral el abanico de datos estadísticos, investigaciones y 
referencias bibliográficas surgidas de examinar cada uno de los ámbitos emergentes seleccionados. No obstante, es posible apuntalar ese panorama volviendo sobre las páginas introductorias.

Una mención aparte merece la tesis que recupera en distintas instancias y que sostiene que el rol de principal cuidadora de la mujer "se conecta directamente con el desarrollo del estado de Bienestar, el diseño de políticas familiares, el vigor de los discursos familistas y el mercado de trabajo" (pág. 44). En contextos de crisis socioeconómicas o de una reducida inversión pública, las redes familiares proveen apoyo y asistencia, especialmente, las mujeres, y se refuerzan los discursos familistas. La profundización del caso español a partir del análisis de la crisis de 2008 y la comparación con otros Estados — en particular los escandinavos-, contribuye a la reflexión sobre cómo el dominio del parentesco se vincula y se ve afectado por otros dominios, tales como la política y el género.

La lectura de este libro merece la pena por varias razones. En primer lugar, vista la enorme producción académica sobre el tema, el autor propone una manera posible de organizar este campo y sistematizar algunos enfoques. En segundo lugar y en relación con ese trabajo, ofrece una guía para rastrear y explorar autores, abordajes y debates sobre el campo de la familia y el parentesco en la actualidad. Finalmente, constituye un interesante ejercicio de aproximación interdisciplinar al estudio de un tema específico que se refleja en la exposición y articulación de investigaciones que responden a diferentes disciplinas - antropología, demografía, sociología, historia, economía, etc.pero también en el manejo de datos estadísticos provenientes de instituciones españolas, extranjeras e internacionales, buscando de esa forma "facilitar la contextualización teórica de estas magnitudes, así como la traducción sociodemográfica y estadística de ciertas tesis o supuestos teóricos" (pág. 21). Por otra parte, la mirada antropológica se expresa cada vez que Grau Rebollo vuelve sobre el problema de las terminologías del parentesco, en particular, los usos y significaciones de las categorías nativas que imponen los nuevos modelos de familia y que obligan también a repensar los conceptos analíticos a través de los cuales nos aproximamos a ellos.

Silvina Smietniansky

Consejo Nacional de Investigaciones Científicas y Técnicas (CONICET). Centro de Estudios en Historia, Cultura y Memoria/ Universidad Nacional de Quilmes (CeHCMe/ UNQ) silvismiet@gmail.com

\section{Referencias bibliográficas}

González Echevarría, Aurora (2010). "Sobre la definición de los dominios transculturales. La antropología del parentesco como teoría sociocultural de la procreación". Alteridades, 20 (39): 93-106.

Needham, Rodney (1971). "Remarks on the Analysis of Kinship and Marriage”, en R. Needham (Ed.), Rethinking Kinship and Marriage. Londres: Tavistock Publications, 1-34.

Schneider, David (1984). A Critique of the Study of Kinship. Ann Arbor: The University of Michigan Press. 